\title{
Iris atrophy in sickle cell disease
}

\author{
R W ACHESON,' S M FORD,' G H MAUDE,' $R$ W LYNESS, ${ }^{2}$ AND \\ G R SERJEANT
}

From the 'Medical Research Council Laboratories (Jamaica), University of the West Indies, Kingston, Jamaica, and the ${ }^{2}$ Departments of Pathology and Ophthalmology, the Queen's University of Belfast

SUMMARY Iris atrophy, of unknown origin and believed to be secondary to the vaso-occlusive process of sickle cell disease, has been observed in 25 eyes of 22 patients (two SS disease, 20 SC disease). The crude prevalence was highest in males with SC disease, in whom $14.7 \%$ of patients were affected. Iris atrophy was closely associated with proliferative sickle retinopathy in the same eye. Analysis of haematological indices failed to reveal any significant differences between patients with and without iris atrophy. The characteristics and distribution of iris atrophy are described as well as the histopathology in one 68-year-old male patient with SS disease.

The vaso-occlusive process characteristic of sickle cell disease produces a variety of lesions in the eye. Although most widely recognised in the conjunctival vessel anomalies and in ischaemia of the retinal periphery with proliferative sickle retinopathy, the iris vessel system may also be affected. Iris atrophy has been previously reported in six eyes of three patients, all with sickle cell-haemoglobin C (SC) disease, ' 2 but no assessments of prevalence have been possible. The screening of large numbers of patients with sickle cell disease in Jamaica has detected 26 patients with iris atrophy, allowing an assessment of the prevalence, risk factors, associated clinical features, and characteristics of this complication.

\section{Material and methods}

The patients attended the Sickle Cell Clinic of the University Hospital of the West Indies, Kingston, Jamaica, and a group of peripheral sickle cell clinics operated by the staff of the Medical Research Council (MRC) Laboratories. During the period 1 January 1984 to 31 May 1985 examination of the iris was performed in 414 patients aged 15 years and over. There were 182 patients ( 75 male, 107 female) with homozygous sickle cell (SS) disease and 232 patients, (116 male, 116 female) with sickle cell-haemoglobin $\mathrm{C}$ (SC) disease. Phthisis bulbi precluded observations on one eye of six patients (one SS male, five SC

Correspondence to Dr G R Serjeant, Medical Research Council Laboratories, University of the West Indies, Kingston 7, Jamaica, WI. males), but the iris was examined in the remaining 822 eyes of 414 patients. All patients with iris atrophy had detailed examinations of the anterior chamber with slit-lamp, gonioscopy, photography and tonometry, and fundus examinations. Fluorescein angiography of the iris was performed in selected cases.

The extent of sectoral iris atrophy was measured circumferentially by clock hours and radially with the collarette and contraction furrows as landmarks. Non-sectoral atrophic patches in the iris were measured as diameter in millimetres. Histological examination of iris atrophy was possible in one patient who died during the course of the study.

\section{Results}

Iris atrophy was present in 31 eyes of 26 patients. In two eyes (both SC females), the iris atrophy occurred as part of the syndrome of anterior segment ischaemia, and in one SS female patient an atypical sectoral iris atrophy was noted in an eye which had previously been operated on for detached retina. In three eyes (one SS male, two SC males), a history of trauma and notching of the pupil with or without angle recession suggested that trauma may have been causally related to the iris atrophy. There remained a group of 25 eyes in 22 patients in whom the likely cause of iris atrophy was a sickle cell related occlusion of the vascular supply of the iris ('spontaneous' iris atrophy).

In this spontaneous group there were two patients with SS disease (one male, one female) and 20 patients with SC disease (17 male, three female). 
Table 1 Crude prevalence rates of 'spontaneous' iris atrophy

\begin{tabular}{|c|c|c|c|c|}
\hline & \multicolumn{2}{|l|}{ Patients } & \multicolumn{2}{|l|}{ Eyes } \\
\hline & $\begin{array}{l}\text { Total } \\
\text { examined }\end{array}$ & $\begin{array}{l}\text { Total } \\
\text { affected }\end{array}$ & $\begin{array}{l}\text { Total } \\
\text { examined }\end{array}$ & $\begin{array}{l}\text { Total } \\
\text { affected }\end{array}$ \\
\hline \multicolumn{5}{|l|}{ SS disease } \\
\hline Males & 75 & $1(1 \cdot 3 \%)$ & 148 & $1(0 \cdot 7 \%)$ \\
\hline Females & 107 & $1(0.9 \%)$ & 213 & $1(0.5 \%)$ \\
\hline \multicolumn{5}{|l|}{ SC disease } \\
\hline Males & 116 & $17(14 \cdot 7 \%)$ & 225 & $20(8.9 \%)$ \\
\hline Females & 116 & $3(2 \cdot 6 \%)$ & 230 & $3(1 \cdot 3 \%)$ \\
\hline
\end{tabular}

*Excluding those with phthisis bulbi and those with iris atrophy probably associated with anterior segment ischaemia or trauma.

Table 2 Age of patients affected by 'spontaneous' iris atrophy

\begin{tabular}{|c|c|c|c|c|}
\hline \multirow{3}{*}{$\begin{array}{l}\text { Age group } \\
\text { (years) }\end{array}$} & \multicolumn{2}{|l|}{ SS disease } & \multicolumn{2}{|l|}{ SC disease } \\
\hline & \multicolumn{2}{|l|}{ Patients } & \multicolumn{2}{|l|}{ Patients } \\
\hline & Examined & Affected $(\%)$ & Examined & Affected $(\%)$ \\
\hline $15-29$ & 87 & $0(-)$ & 136 & $2(1 \cdot 5)$ \\
\hline $30-44$ & 69 & $0(-)$ & 70 & $13(18 \cdot 6)$ \\
\hline $45+$ & 26 & $2(7 \cdot 7)$ & 26 & $5(19.2)$ \\
\hline
\end{tabular}

The crude prevalence rates (Table 1) confirm the complication to be most common in males with SC disease. The age of onset was unknown, since in none of the 'spontaneous' cases was the onset of iris atrophy prospectively recorded, but the complication appeared to be more common with advancing age (Table 2).

Iris atrophy affected the right eye in 12 patients, the left in seven, and was bilateral in three (all SC males), the difference in right/left distribution failing to reach significance at the $5 \%$ level. The pattern of iris atrophy was confined to sectoral involvement limited to the pupillary margin in two eyes (type 1), extending radially from the pupillary margin to the collarette (type 2) in nine eyes, and to the contraction furrows (type 3 ) in nine eyes. Atrophy was confined to circumscribed areas measuring 1-4 mm diameter in the ciliary zone in four eyes. One patient had both a type 3 radial involvement from 9 to 1 o'clock and a separate lesion in the ciliary zone at 50 'clock (Fig. 1). The extent of circumferential involvement by sectoral lesions varied from $30^{\circ}$ to $180^{\circ}$ with a median of $90^{\circ}$. The 21 sectoral lesions affected predominantly the temporal side in 16 eyes and the nasal side in five eyes, the difference being significant at the $5 \%$ level. Of the five ciliary lesions four occurred in nasal sectors and one at 6 o'clock.

Atrophic areas appeared thinned and flat with loss

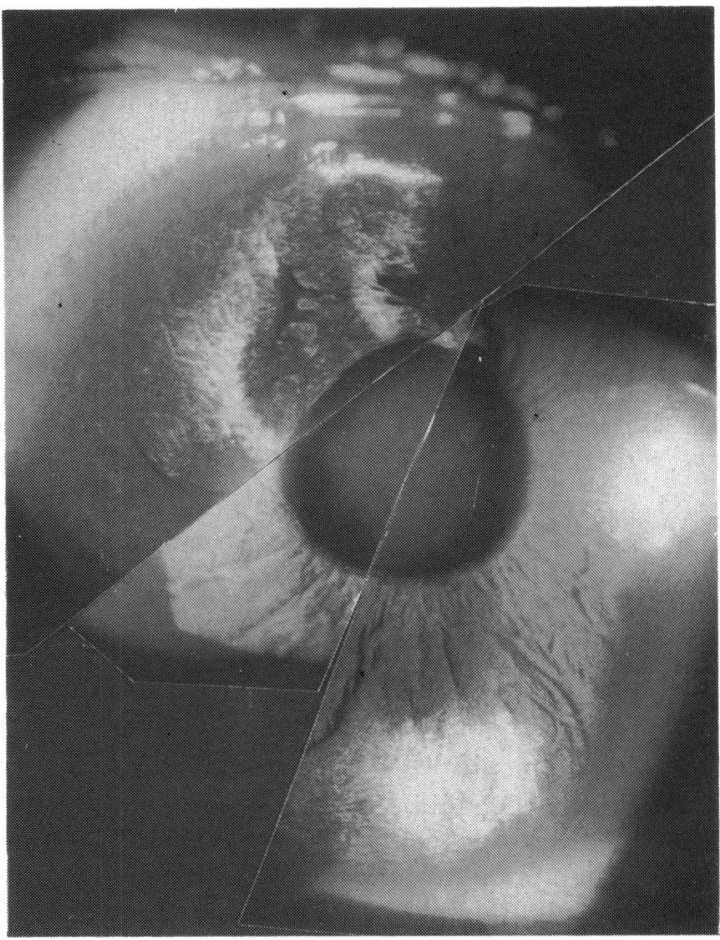

Fig. 1 Composite photograph of iris atrophy in the right eye of a 43-year-old male with SC disease demonstrating both sectoral iris atrophy at 9 to $10^{\circ}$ clock and a circumscribed area in the ciliary zone at $50^{\circ} \mathrm{clloc}^{\circ}$.

of the normal folds of stromal tissue (Fig. 2), and appeared to affect predominantly the anterior border layer and stromal tissue. In sectoral lesions the pupillary margin was always affected, with loss of the normal pupillary frill and occasionally rupture of the pupillary margin (Fig. 3). When retroilluminated the

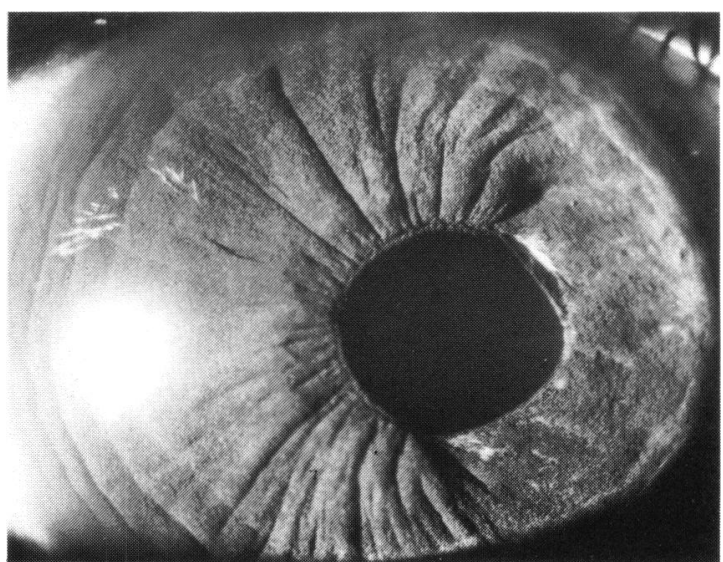

Fig. 2 Sectoral iris atrophy from I to 6o clock in the nasal side of the right eye in a 25 -year-old male with SC disease. 


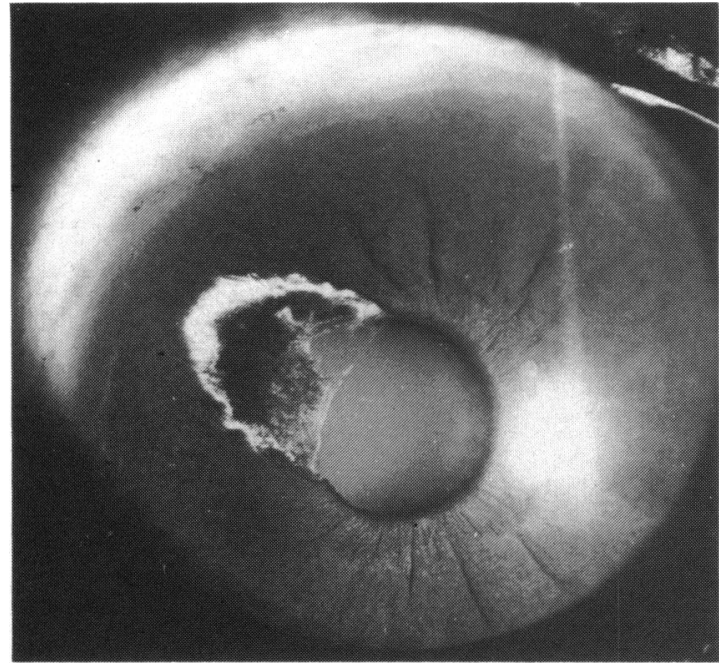

Fig. 3 Sectoral iris atrophy from 8 to $120^{\circ}$ clock in the nasal side of the left eye in a 46-year-old female with SS disease.

atrophic areas could be seen to be thin and translucent with full thickness holes. Pigmentary changes were common in atrophic areas, hyperpigmentation occurring occasionally but most areas being mottled with pale white fibres coursing radially (Fig. 4). The atrophy never extended peripheral to the contraction furrows, and the anterior chamber angles were normal and open, though eyes with iris atrophy frequently showed more pigment, especially in the inferior angle, when compared with eyes without iris atrophy. Sphincter function was affected in some patients.

Fluorescein angiography of the iris in nine eyes

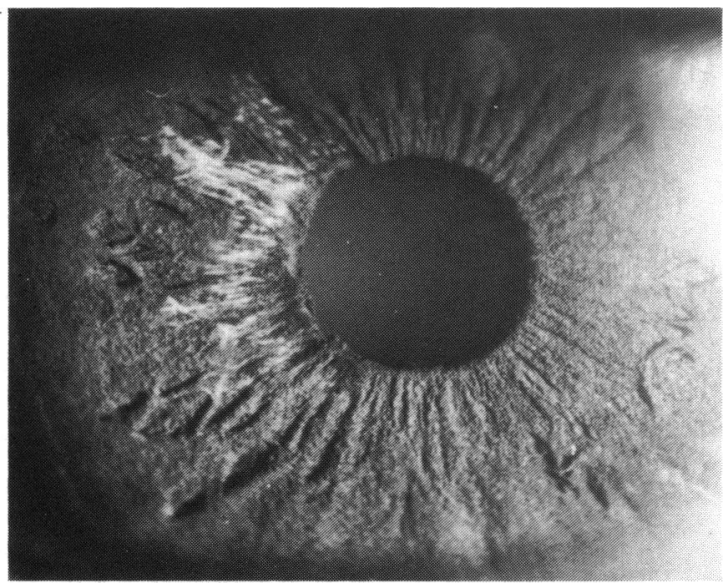

Fig. 4 White fibres in sectoral area of iris atrophy from 8 to $110^{\prime}$ clock in the temporal side of the right eye in a 56-year-old female with SC disease.

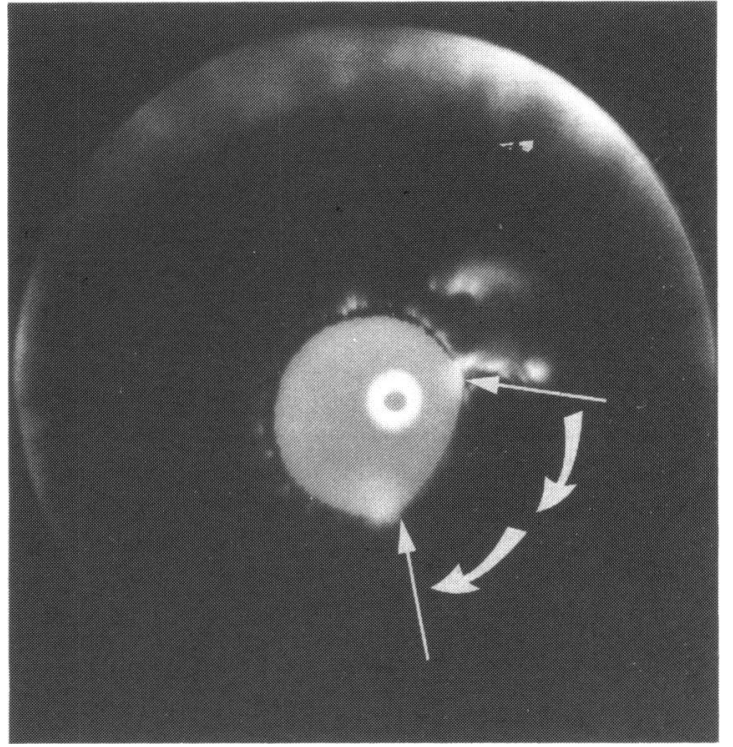

Fig. 5 Fluorescein angiogram of iris in the left eye of a 59 year-old male with SC disease. White arrows mark extent of sectoral iris atrophy.

(seven patients) confirmed that the atrophic areas were avascular. Leakage of fluorescein was generally confined to the margin of the atrophic areas (Fig. 5), but occasionally a spotty leakage was observed over the whole surface of the iris (Fig. 6).

The histopathology of iris atrophy was studied in a 68-year-old male with SS disease in whom a sectoral

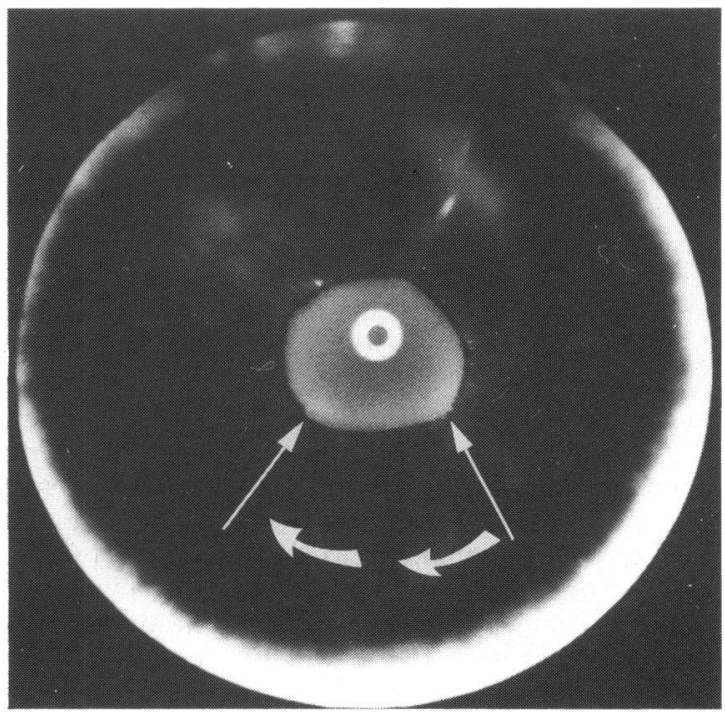

Fig. 6 Fluorescein angiogram of iris in the right eye of a 42 year-old male with SC disease showing diffuse spottyleakage. White arrows mark the extent of sectoral iris atrophy. 


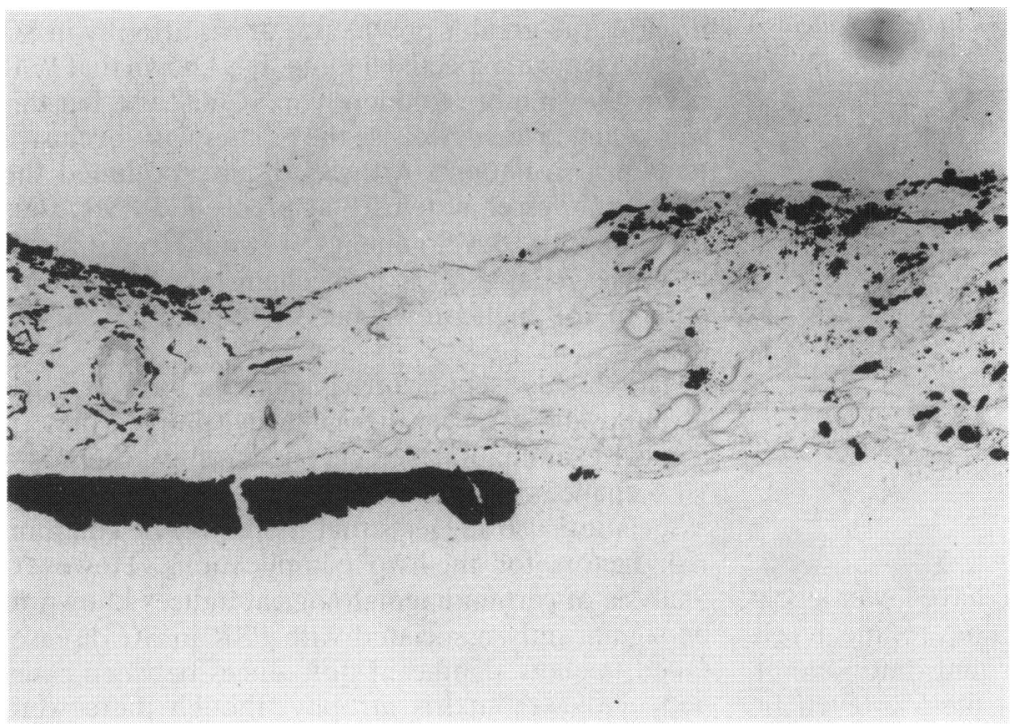

Fig. 7 Section through full-thickness infarct of iris stroma in a 68 year-old male with SS disease. The detachment of pigment epithelial layer to the right side of the figure was artefactual. (Haematoxylin and eosin, $\times 5())$.

necrosis from 9 to 12 o'clock extended from the pupillary margin to the contraction furrows of the right eye. Pathological examination showed necrosis between the midzone of the iris and the pupillary margin, generally affecting the anterior part of the stroma, though there was a full-thickness infarction of the iris at one site (Fig. 7). The dilator and sphincter pupillary muscles were involved by infarction and atrophy was visible adjacent to the infarcted area. Areas of infarction were seen at the root of the iris. The pigment epithelium was generally intact and appeared to be spared by the infarctive process, but pigment from necrotic melanocytes in the stroma was dispersed and removed from infarcts at later stages. There was focal ischaemic necrosis and atrophy with coagulative necrosis of blood vessels and stroma. Endothelial cell nuclei failed to stain, leaving ghosts of blood vessels amid dissociated wisps of stromal fibres. Blood vessels in the ciliary body contained sickled red blood cells, but there was no evidence of organised thrombus, vessel wall thickening, or of an active inflammatory process.

The preponderance of iris atrophy among SC males raises the possibility of an association with proliferative sickle retinopathy (PSR), which is also recognised to be most common in this group. This analysis was confined to male SC patients in whom 212 eyes were observed for both iris atrophy and PSR. Iris atrophy occurred in 19\% of eyes affected by PSR but was observed in only $2 \%$ of eyes without PSR (Table 3 ), the difference being highly significant. Of the 20 eyes with iris atrophy 18 had PSR in the same eye and two had PSR in neither eye. Since many of the patients with PSR had received photo- coagulation therapy with either the xenon arc or argon laser, it was possible that the high prevalence of iris atrophy in PSR patients was related to therapy. Further analysis of the prevalence of iris atrophy in those with PSR with photocoagulation $(18 \%$ iris atrophy) and without photocoagulation $(20 \%)$ revealed no difference between the groups (MantelHaenszel $\chi^{2}=(0 \cdot 0)(03$, not significant) indicating that photocoagulation did not appear to increase the chance of developing iris atrophy.

A study of the haematological risk factors for iris atrophy was performed by comparing by Student's $t$ test the distribution of haematological indices in those with and without iris atrophy. In order to minimise sex and age related differences this study was confined to male patients with SC disease aged 30 years and over and utilised the most recent steady state data for each patient (mean $2 \cdot 2$ years earlier, range $0-7$ years). The results (Table 4 ) indicate that

Table 3 Prevalence of iris atrophy in SC male eyes with and without PSR

\begin{tabular}{|c|c|c|c|c|c|c|}
\hline \multirow[t]{3}{*}{ Age group } & \multicolumn{3}{|c|}{ PSR positive } & \multicolumn{3}{|c|}{$P S R$ negative } \\
\hline & \multicolumn{3}{|c|}{ Iris atrophy } & \multicolumn{3}{|c|}{ Iris atrophy } \\
\hline & + & - & $\%+v e$ & + & - & $\%+v e$ \\
\hline $15-29$ & 2 & 36 & 5 & 0 & 86 & () \\
\hline $30-44$ & 12 & 31 & 28 & 2 & 22 & 8 \\
\hline $45+$ & 4 & 10 & 29 & 0 & 7 & 0 \\
\hline All & 18 & 77 & 19 & 2 & 115 & 2 \\
\hline
\end{tabular}

Mantel-Haenszel $\chi^{2}$ allowing for age groups $=7 \cdot 29, p<0 \cdot(01$. 
Table 4 Some haematological indices in male SC patients with and without iris atrophy

\begin{tabular}{|c|c|c|c|c|c|}
\hline & \multicolumn{2}{|c|}{$\begin{array}{l}\text { Iris atrophy } \\
(n=15)\end{array}$} & \multicolumn{2}{|c|}{$\begin{array}{l}\text { No iris atrophy } \\
(n=34)\end{array}$} & $\begin{array}{l}\text { Significance } \\
t\end{array}$ \\
\hline & Mean & $S D$ & Mean & $S D$ & \\
\hline $\log (\mathrm{HbF}+4)$ & $1 \cdot 56$ & $0 \cdot 14$ & 1.62 & $0 \cdot 17$ & $-1 \cdot 14 \mathrm{NS}$ \\
\hline $\mathrm{Hb}$ & $13 \cdot 3$ & $1 \cdot 3$ & $12 \cdot 7$ & $1 \cdot 3$ & $1.58 \mathrm{NS}$ \\
\hline $\mathrm{MCHC}$ & $35 \cdot 7$ & $1 \cdot 7$ & $35 \cdot 6$ & 1.9 & $0 \cdot 18 \mathrm{NS}$ \\
\hline MCV & $88 \cdot 6$ & $7 \cdot 1$ & $84 \cdot 3$ & $7 \cdot 2$ & $1.93 \mathrm{NS}$ \\
\hline $\log ($ reticulocytes+1) & 1.67 & $0 \cdot 36$ & $1 \cdot 41$ & 0.47 & 1.94 NS \\
\hline
\end{tabular}

NS $=$ not significant at the $5 \%$ level

$\mathrm{MCHC}=$ mean corpuscular hacmoglobin concentration . $\mathrm{MCV}=$ mean cell volume

patients with iris atrophy tended to have lower levels of fetal haemoglobin and higher values for mean cell volume (MCV) and reticulocyte count, but none of the differences were significant at the $5 \%$ level. In view of the strong relationship between iris atrophy and PSR, and of the previously established relationship of PSR with higher MCV and lower HbF, the lack of significant relationships with iris atrophy was surprising but probably simply reflected the small size of the study group.

\section{Discussion}

Iris atrophy has been previously reported in only three patients with sickle cell disease,${ }^{12}$ giving the impression that this is an uncommon finding. The current report describing 31 affected eyes indicates that it is relatively common. Some of these cases have well recognised causes such as anterior segment ischaemia; involvement of a single eye in a female with SS disease was excluded from the series, since it may have been secondary to retinal detachment surgery. Trauma is also a well documented cause, and three further cases were excluded as being possibly attributable to this. All had a history of moderate trauma, all had notching of the pupil one showed mild angle recession, but none had evidence of radial splits or iridodialysis. None of the remaining cases had evidence of other known causes such as quinine $^{3}$ or herpes zoster, ${ }^{4}$ and there remained a group of 25 eyes in 22 patients who were presumed to have 'spontaneous' iris atrophy associated with sickle cell disease.

This finding is reported for the first time in two patients with SS disease but is most common in male patients with SC disease, occurring in nearly $15 \%$ of this group. This prevalence is probably broadly representative of the patients at risk, though may be affected by a variety of selection biases. Since vasoocclusion is believed to occur more frequently in SS disease, the greater prevalence of iris atrophy in SC disease is an unexpected finding. It is known that PSR occurs much more frequently in SC disease, but this was believed to be because the greater vaso-occlusive tendency in patients with SS disease occluded the abnormal vessel systems that preceeded PSR, thus inhibiting its development. It would be difficult to envisage how such a mechanism could contribute to the high prevalence of iris atrophy in SC disease.

The highly significant association between iris atrophy and PSR in the same eye imples that, if clinical examination detects iris atrophy, there is a $90 \%$ chance of PSR occurring in the same eye. This association also suggests that there may be common risk factors for the two complications. However, analysis of certain haematological indices known to be significantly associated with PSR in SC disease ${ }^{5}$ failed to show significant differences between cases with and without iris atrophy, though there were similar trends, and the lack of significance may have simply reflected the small numbers available for analysis.

The mechanism of vaso-occlusion in the iris is not understood. Although macroscopically the infarcted areas did not appear to extend peripheral to the contraction furrows, the histopathology in the one examined case showed areas of necrosis extending to the root of the iris. The blood supply of the iris is derived from a greater arterial circle in the root of the iris, radial vessels coursing towards the pupillary margin, and a minor arterial circle at the level of the collarette. The area between the collarette and the pupillary margin has the greatest density of fine vessels, and yet this is the region most frequently affected by infarction. Studies of iris circulation by fluorescein angiography in patients with blue irides ${ }^{6}$ have indicated that iris vessels fill more slowly than those in the retina or choroid and that the central part of the iris fills much more slowly than the root, the nasal vessels filling before the temporal vessels. These observations are compatible with the observed pattern of iris atrophy in these patients, which affected predominantly the pupillary margin and central areas, and the temporal side more frequently than the nasal. Unfortunately fluorescein angiography in patients with brown irides is much less informative because of pigment in the iris stroma, and only poor vessel detail is available. A patient studied by Galinos et al.' had a peripupillary neovascular network and a vascular formation resembling a sea fan, though the patient also had diabetes mellitus, suggesting an alternative aetiology for these changes. In the present study fluorescein angiography of the iris in nine eyes revealed almost no vessel details, though atrophic areas appeared to be 
avascular, and spotty leakage of dye from clinically unaffected areas suggested a more widespread vessel abnormality.

Much remains to be learnt about the factors contributing to iris atrophy in sickle cell disease. Although this complication is of limited functional significance, the mechanisms involved may be instructive for the understanding of vaso-occlusion elsewhere in sickle cell disease.

We thank Mr R J Marsh, FRCS, of the Western Ophthalmic Hospital, London and Mr T A Gardiner, MSc, of the Department of Ophthalmology, Queen's University of Belfast, for valuable discussion.

\section{References}

1 Galinos S, Rabb MF, Goldberg MF, Frenkel M. Hemoglobin SC disease and iris atrophy. Am J Ophthalmol 1973; 75: 421-5.

2 Chambers J, Puglisi J, Kernitsky R, Wise GN. Iris atrophy in hemoglobin SC disease. Am J Ophthalmol 1974; 77: 247-9.

3 Knox DL, Palmer CAL, English F. Iris atrophy after quinine amblyopia. Arch Ophthalmol 1966; 76: 359-62.

4 Marsh RJ, Easty DL, Jones BR. Iritis and iris atrophy in herpes zoster ophthalmicus. Am J Ophthalmol 1974; 78: 255-61.

5 Hayes RJ, Condon PI, Serjeant GR. Haematological factors associated with proliferative retinopathy in sickle cell-haemoglobin C disease. Br J Ophthalmol 1981; 65: 712-7.

6 Hayreh SS, Scott WE. Fluorescein iris angiography. I. Normal pattern. Arch Ophthalmol 1978; 96: 1381-9.

Accepted for publication 5 November 1985. 